In questa bozza di testo per il libro „La Tierra del Quetzal - Zentralamerika heute “, che apparirà verso la fine dell'anno per i tipi della Casa Editrice Kümmerly \& Frey, vien (descritto il recarsi della gente al mercato e alla chiesa) di Santo Tomás Chichicastenango nel Guatemala.

\title{
DIE CANADISCHEN PRÄRIEPROVINZEN IM INDUSTRIELLEN UMBRUCH
}

\author{
ERNST WINKLER
}

Mit 1 Tafel und 7 Abbildungen

\begin{abstract}
Die sogenannten drei Prärieprovinzen von Canada: Manitoba (Man.), Saskatchewan (Sask.) und Alberta (Alta.) galten bisher als der Getreidespeicher dieses Staates und darüber hinaus als eine der größten und bedeutendsten Kornkammern der Erde überhaupt. Und zweifellos werden sie diesen Ruf auch in der nächsten Zukunft zu verteidigen wissen. Allein in den letzten Jahren spielten sich in dieser ausgesprochensten Agrarregion Canadas (und vielleicht des ganzen Erdteils) Ereignisse ab, die sie über kurz oder lang zum Gebiet nicht weniger wichtiger Industrien machen werden. Damit wird auch ihr landschaftliches Gesicht mutmaßlich bemerkenswerte Umformungen erfahren. Den im Zuge befindlichen Umbruch zu verfolgen, dürfte daher auch für die Geographie von Interesse sein. Es soll deshalb im folgenden versucht werden, hierzu einen Beitrag zu leisten.
\end{abstract}

\section{„BROAD HORIZONS“}

Den landschaftlichen Grundzug der nahezu 2 Millionen km² (Manitoba 652 218, Saskatchewan 651876 , Alberta $661161 \mathrm{~km}^{2}$ ) großen (also einen Fünftel der Fläche Canadas umfassenden) Prärieregion zeichnet der Wechsel weiter, welliger bis flacher Plateaus und Ebenen und zahlreicher tief in sie eingeschnittener breiter Täler, deren im ganzen gering erscheinende «Reliefenergie» dem Gebiet die Namen Plains», «Great Plains », «Interior Plains oder Lowlands » eingetragen hat. Diese Namen spielen auf eine Einheitlichkeit an, welche den Provinzen keineswegs eignet, zumal auch nur ein relativ kleiner Bereich, kaum ein Drittel der Gesamtfläche, von der Vegetation eingenommen ist, die ihre Flachheit besonders zu betonen vermöchte: von Steppe, da vielmehr Wälder und Parklandschaften den Hauptteil des Mittelwestens beanspruchen. Die Prärien, dieses «great inland empire» stellen so eine kaum weniger mannigfaltige Großlandschaft ${ }^{1}$ Canadas dar als dessen übrige Hauptgebiete, und diese konstitutionelle Eigenschaft findet in der Disposition, in der Eignung für menschliche Besiedlung und Nutzung ein Korrelat, das bisher nur teilweise gewerte wurde. Denn der Mittelwesten ist nicht nur - wie er bisher vor allem beurteilt wurde - ein klimatisch wie pedologisch und geomorphologisch bemerkenswert gut disponierter Agrarraum: er besitzt nicht allein in weithin verbreiteten Schwarz- und Braunerden vorzügliche und dank der vorwiegenden Flachheit des Geländes leicht pflügbare Nährgründe und ein zwar herbes, trockenes, aber im ganzen genügend (und jahreszeitlich gut verteilte) Niederschläge (Sommerregen) empfangendes Klima. Der Untergrund repräsentiert, wie sich immer mehr heraus-

${ }^{1}$ Allein hinsichtlich der „naturräumlichen “ Gliederung ließen sich gestützt auf die starke Durchtalung der Prärie-, ,Tafel “ und die dadurch bedingte Differenzierung von Topographie, Hydrographie, Regionalklimatologie und Vegetation zahlreiche "Naturlandschaftsindividuen " verschiedener Größenordnung - so an die 1000 von der Größe eines mittleren Schweizer Kantons $\left(2000 \mathrm{~km}^{2}\right)$, 10000 von der Größe einer mittleren Schweizer Tallandschaft $\left(200 \mathrm{~km}^{2}\right)$ - sondern, die trotz ihrer vielfachen Analogie zweifellos ebenso viele Eigentümlichkeiten aufweisen. Auch ein fü̈chtiger Blick wie er dem Verfasser mit seinem Freund Prof. Dr. H. BerNhard 1948 in verschiedenen Gegenden der Prärieprovinzen: so um Winnipeg, um Regina, um Edmonton, im Peace River Gebiet und um Calgary innerhalb von etwa 3 Wochen intensiver Fahrten vergönnt war - vermag von der hohen landschaftlichen Variabilität der in der Regel als eintönig geschilderten Prärienregion zu überzeugen. 


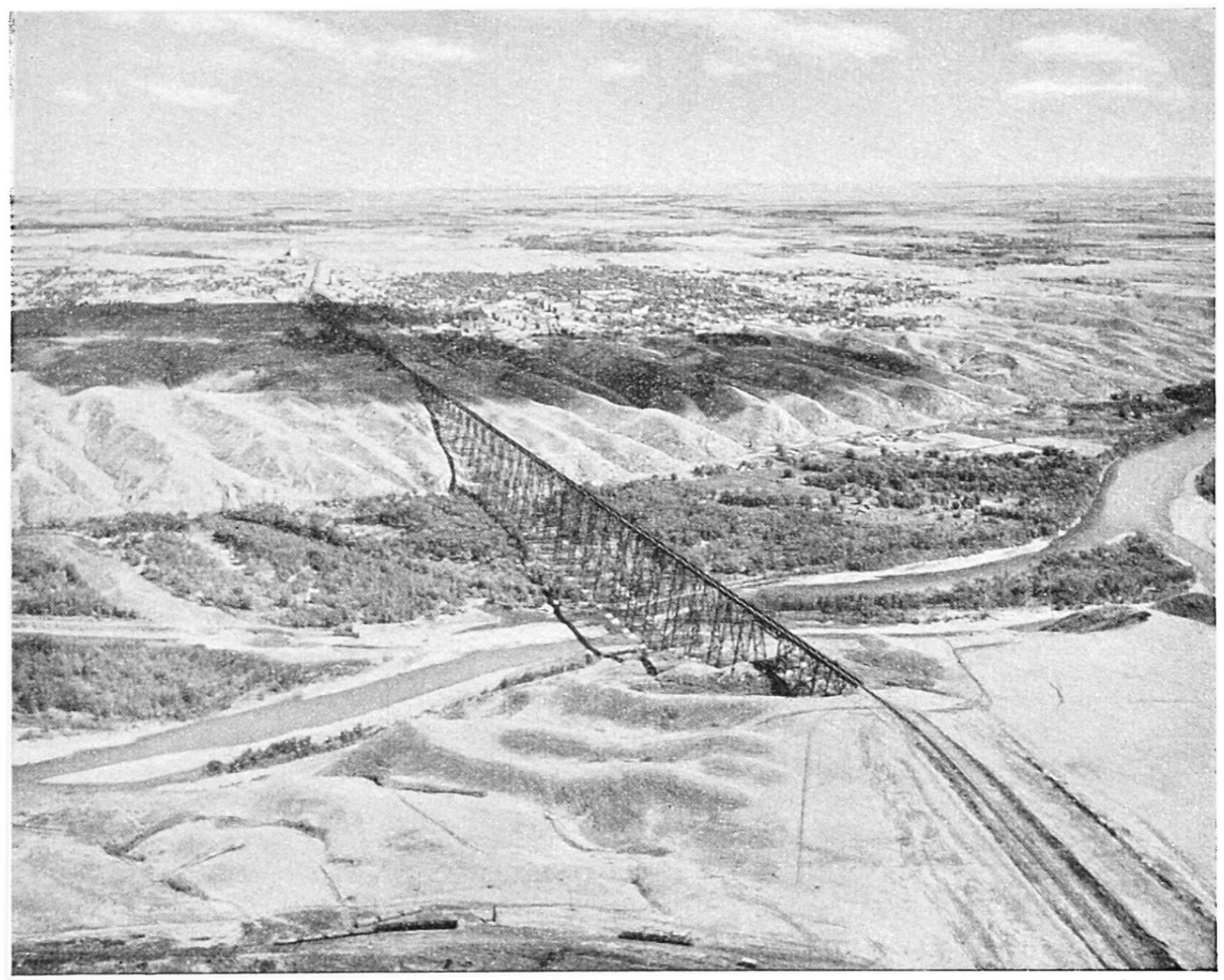

Lethbridge, Südalberta. Blick auf die aride Hochprärie, in der, z. T. mittelst bedeutender Bewässerung, die Stadt Lethbridge als Standort von Müllereien und Rübenzuckerfabriken sich entwickeln konnte. Photo Canadian Pacific Railway.

stellt, auch eine Quelle mannigfacher Mineralschätze, die die Prärien zum Standraum nationalpolisch wie gesamtwirtschaftlich bedeutsamster Montanwirtschaft und damit künftiger Industrien stempeln. Dies alles zusammengenommen läßt verstehen, daß J. Gislason sein impulsreiches «Prärie Panorama» mit den Worten einleiten konnte «'The prairie horizon is a broad horizon. Clear skies and great expanses of unobstructed view have acustomed the Westerner to a large perspective. He sees beyond the limits of his own acres, beyond his neighbour's lands and farther yet - by exercise of some imagination - he sees the whole world ». Die Symbolik dieser Sätze umreißt keineswegs nur die weiten Horizonte der Prärientopographie; sie betont auch die Tatsache, daß Topographie und landschaftliche Substanz von jeher eine großzügige Lebensauffassung, das Bewußtsein, einen großen «Job» in diesem Lande der unabsehbaren und reichen Räume machen zu können, im Menschen geweckt und wach gehalten haben, das in der breiten Natur des Westerners ebenso zu prägnantem Ausdruck gelangt wie in seinen Aktionen, die je länger desto kühner werden.

In der Besiedlungs- und Kulturlandschaftsgeschichte Canadas spielten die heutigen Prärieprovinzen allerdings lange nur die Rolle eines Durchzugsgebietes nach den aussichtsvoller erscheinenden Hochgebirgsgegenden des Westens und zur Pazifikküste, und bis ins 19. Jahrhundert hinein begnügten sich die Machthaber im Lande zur Hauptsache damit, diese Gebiete durch zahlreiche Forts gegen die halbnomadischen, teils feldbauenden, teils jagenden und fischenden Prärieindianer zu sichern. Politisch in Beschlag genommen war die Region freilich schon im 17. Jahrhundert, seitdem der englische König, Karl II. 1670 alles Land westlich der Hudsonbai der „Company of Adventurers of England Trading into Hudson's Bay (HBC) “ überantwortet hatte. Doch blieb die nach dem ersten Gouverneur, Prinz Rupertsland genannte Region bis zum Zeitpunkt ihres Verkaufs an die 
canadische Regierung (1869), so gut wie ausschließlich als Pelzquelle genutzter Kolonialbereich, wenn auch inzwischen mehrfache Versuche gemacht worden waren, aus dem Land der unermeßlichen Wälder, Steppen und Halbwüsten, der Indianer, Büffel, Elche, Wapitis, Bären und Biber, der kurzen Sommer und harten, langen, schneesturmdurchbrausten Winter ein Siedlungsland zu machen. Die beiden historischen Daten waren der Kolonisationsversuch des schottischen Lords SEI.KIRK am Red River 1811-15, der anfänglich scheiterte, jedoch die HBC dazu veranlaßte, das von SELKIRK erworbene Land zurückzukaufen und selbst die Ansiedlung von Kolonisten in die Hand zu nehmen, während sie vordem deren schärfste Gegnerin gewesen war - sodann die ebenso berühmte wie berüchtigte Expedition Palliser 1857, die im Auftrag der britischen Regierung die agrarischen Möglichkeiten des Prärienlandes zu erforschen hatte und zum Schluß kam, daß es für eine landwirtschaftliche Besiedlung ungeeignet (unfit) sei. Gleichzeitige analoge Gutachten von Dawsox, HisD u. a. hinderten indes die Regierung nicht am Rïckkauf aller Ländereien von der HBC und am Versuch der Erschließung, da in Ostcanada das siedlungsfähige Land damals bereits vergeben war. Damit und vor allem durch die Schaffung einer ersten Prärienprovinz im Jahre 1870, der (im Vergleich mit heute freilich wesentlich kleineren) Provinz Manitoba, sowie der ihrer Regierung unterstellten aus dem Rest des Nordwestterritoriums gebildeten vier Distrikte Assiniboia, Saskatchewan, Alberta und Athabasca war die Entwicklung der Prärie eingeleitet. Es zeigte sich bald, daß die getroffenen Maßnahmen für das ganze Land von entschiedenem Vorteil waren. Immerhin machte trotz des 1872 nach dem Muster der USA erlassene Heimstädtengesetzes (Homestead Law), das dem Einwanderer erhebliche Vorteile gewährte, sowie der Landesvermessung nach dem Townshipsystem, die Besiedlung im folgenden Jahrzehnt nur wenige Fortschritte. Einerseits erkannten die Kolonisten die agrarischen Vorteile der Prärie nicht, andrerseits fehlten damals mangels geeigneter Verkehrswege und Transportmittel auch noch die Absatzmöglichkeiten. Den ersten Aufschwung veranlaßte die Niederlassung osteuropäischer (rußlanddeutscher) Menoniten im Gebiete von Winnipeg ums Jahr 1874 und der Bau der Canadian Pacific Railivay 1878-1885, die den Mittelwesten verkehrstechnisch aufschloß. Während die Mennoniten als mit der Produktivität und Eigenart der Steppe vertraute Bauern die Pioniere der Agrarisierung der Prärien und ihrer allmählichen Besiedlung wurden, indem ihre erfolgreichen Anbauversuche in der Folge weitere Ackerbauer aus aller Herren Länder, aus Ostcanada, Mittel-, West-, Ost- und Nordeuropa anlockten, wirkte die Bahn als eigentliche "Conditio sine qua non " der Erschließung überhaupt und als „Verstärkerin “, insofern mit ihr sowohl der Siedlerzustrom gesteigert als namentlich nun auch die Produktion der neuen Agrargebiete nutzbringend gelenkt werden konnte. Wenn dennoch 'die Besiedlung der Prärien erst nach 1900 in schnelleres Rollen kam, die Bevölkerung damals erst rund 420000 (8\% der Gesamteinwohnerschaft Canadas erreicht hatte, während sie 1871 freilich erst rund $25000(0,7 \%$ der Gesamtbevölkerung des Landes) zählte, so wohl in erster Linie, weil auch bis dahin noch zahlreiche Anlaufsschwierigkeiten wie Rodung weiter Gebiete, oftmalige Ernteeinbußen, zu geringe Bahndichte, Absatzhemmnisse, Mangel an Arbeitskräften usw. zu überwinden waren. Um die Jahrhundertwende stand jedoch fest, daß die Prärien Canadas ein Zukunftsland erster Ordnung seien. Offizielle Propagandaführer, Presse und Schriften sprachen damals schon von der „zukünftigen Kornkammer des Britischen Weltreiches ", von einem Gebiete, das, nach des Parlamentariers Sir R. Cartwright Meinung „Raum für eine Bevölkerung von mindestens 50 Mill. Seelen “ habe. Dabei wurde keineswegs allein nur auf die ackerbaulichen Werte des Mittelwestens abgestellt. Die zeitgenössischen Quellen weisen auch darauf hin, daß dort „die schönsten Wälder der Welt, unerschöplliche Quellen von Eisen und Kohlen, Gold und Silber... prächtige Weiden und unbegrenzte Mengen natürlichen Heues lägen, um die feinsten Typen von Tieren, Pferden, Rindern und Schafen hervorzubringen “. Die Regierung trug denn auch solchen Urteilen und Prognosen dadurch Rechnung, daß sie 1905 zwei weitere Provinzen: Saskatchewan und Alberta, schuf und 1912 Manitoba bis zum 60 Breitengrad (von 190966 auf gut $650000 \mathrm{~km}^{2}$ ) erweiterte, womit die Mitte Canadas auch territorialpolitisch konsolidiert war.

Die zweite bedeutungsvolle Aera der Prärien, die Aera als Weizenland hatte damit begonnen.

$$
\text { „WHEAT IS KING - WHEAT HAS BEEN KING “ }
$$

Um 1880 belief sich die Weizenanbautiäche der Prärieprovinzen auf rund 57000 acres ( 1 acre $=0,4047$ ha), auf́ der insgesamt 1,1 Mio bushels Weizen ( 1 bushel $=27,22 \mathrm{~kg}$ ) geerntet wurden. Zu Beginn des 20. Jahrhunderts, 1901, war die Anbaufläche auf 2517000 acres, der Ertrag auf 63,3 Mio bushels angestiegen und in der Folge fand eine weitere Eriveiterung des Weizenareals statt, das 1921: $21591000 ; 1931$ : 25345000 und 1948: 23045000 acres betrug. Die entsprechenden Erträge bezifferten sich auf 293,4 Mio bushels im Jahre 1921, 286,0 Mio 1931 und 520 Mio bushels im Jahre 1951. Diese Erträge, die nicht den Höchststand bezeichnen (1928: 560 Mio bushels), entsprachen seit etwa 1910 zwischen 90 und $95 \%$ der gesamtcanadischen Produktion, woraus die hervorragende 


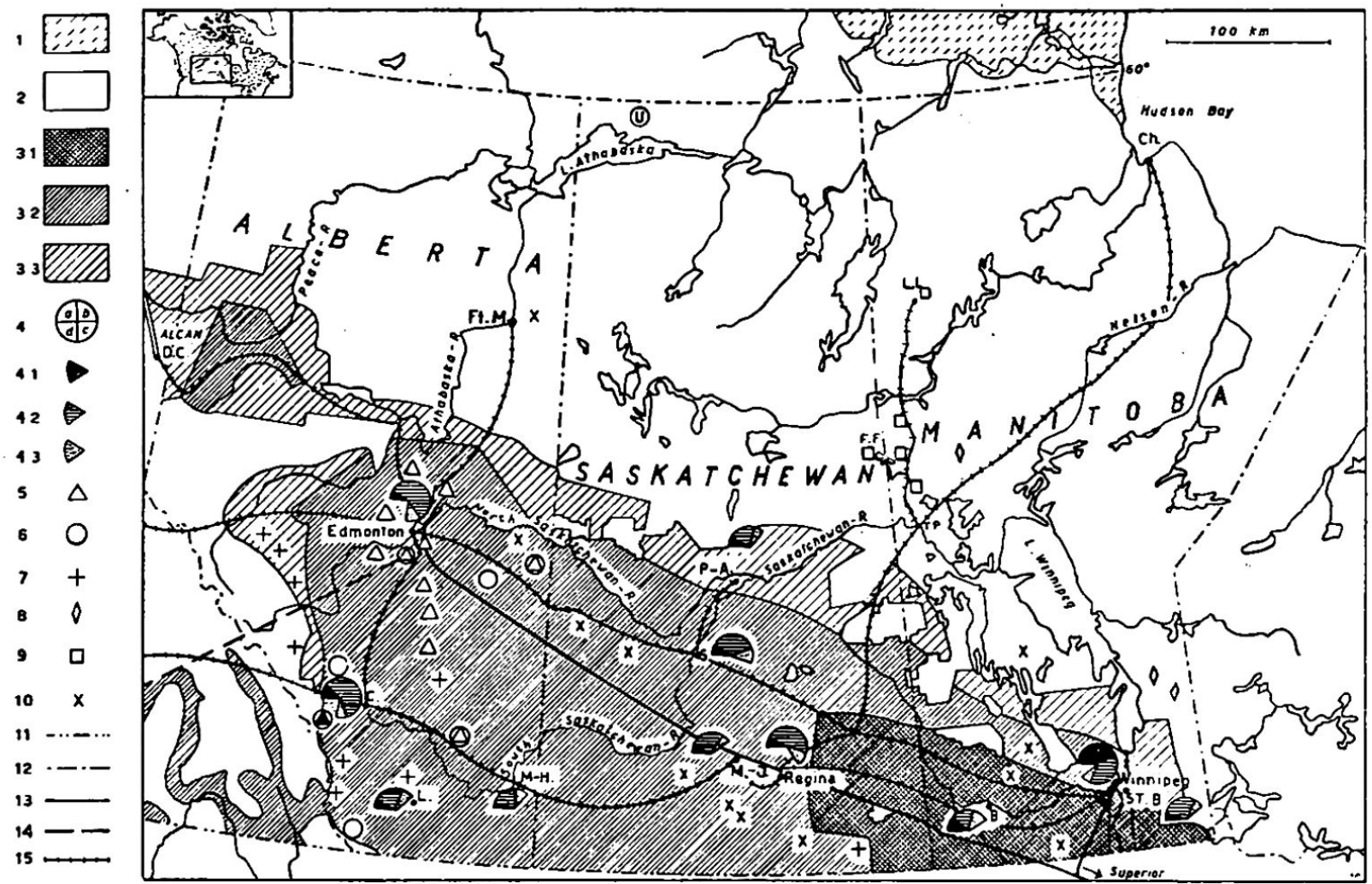

Skizze der Kulturlandschaftsentwicklung der canadischen Präriepro.vinzen 1900-1950.

1 Tundren, 2 (vorwiegend) Wälder, 3 agrarisches Nutzland $31: 1900,32: 1926,33: 1950(51), 4$ wichtigste Städte : a $0-10000$, b $10-50000$, c 50-100 000, d über $100000(100-300000), 41: 1901$, 42: 1926, 43: 1951, 5 Erdölfelder (schwarz Stand um 1925, weiß: 1950), 6 Erdgasfelder, 7 Kohle, 8 Gold, 9 Kupfer-Gold-Zink (Nickel), 10 Salze, U Uran, 11 Staatsgrenze, 12 Provinzgrenzen, 13 bestehende Pipelines, 14 im Bau befindliche Pipeline (Trans-Mountain Pipeline), 15 Eisenbahnen, ALCAN: Alaska Higway (1942), DC. Dawson Creek, Ft. M Fort McMurray, PA Prince Albert, FF Flin Flon, TP The Pas, Ch Churchill, C Calgary, L Lethbridge, MH Medicine Hat, MJ Moose Jaw, B Brandon. St. B. St. Boniface, LL Lynn Lake. Nach Canada Year Book.

Stellung der Prärieprovinzen ersichtlich wird, die den größten Teil des auf 20 bis $-30 \%$ bezifferten canadischen Anteils am Welthandel trugen. Anfänglich beschränkte sich die Hauptproduktion auf Manitoba, das noch um die Wende des 19. Jahrhunderts über $70 \%$ des westcanadischen Weizens lieferte. Schon 1908 überflügelte jedoch Saskatchewan die Nachbarprovinz und vermochte seitdem, dank ihren ausgezeichneten Anbaubedingungen, ihren Vorrang zu halten. Mit dem Jahre 1917 rückte Alberta an zweite Stelle unter den mittelwestlichen Provinzen, und sie behielt sie auch in der Folge bei. Wenn bei dieser Schwergewichtsverlagerung von E nach W auch in erster Linie die südlichen Gebiete der Provinzen, der sogenannte eigentliche fertile belt, in Kultur genommen wurden, was sowohl den pedologischen als okkologischen Yerhältnissen entsprach (nur im Süden fand sich offenes Land, während die Mitte und der Norden Waldland darstellen), so fand doch im Laufe der letzten Jahrzehnte (namentlich gegen die Jahrhundertmitte hin) auch eine Ausbreitung des Weizen- und Kulturlandes nach $\mathrm{N}$, insbesondere nach $\mathrm{NW}$ statt. Dabei erlebte vor allem die Gegend des mittlern und obern Peace River im Zuge der Agrarisierung des Mittelwestens eine bemerkenswerte Blüte durch Pioniere aus Ost- und Nordeuropa.

Der hauptsächlich der günstigen Konjunktur im ersten Weltkrieg zu verdankende Aufschwung der Weizenproduktion blieb jedoch nicht ohne Rückschläge. Ganz abgesehen davon, daß viel Boden mit $W$ eizen bestellt wurde, der hiefür ungeeignet war, traten, namentlich in den dreißiger Jahren, aber auch schon vorher (z. B. 1924 und 1929), Dürrekatastrophen auf, die die Weizenerträge stark zurückgehen ließen. Schlimmer wohl als diese Naturrisiken, die in der Folge wenigstens 
teilweise durch umfangreiche (gegenwärtig gegen 900000 acres, d. h. ca. 350000 ha erfassende) Irrigationsanlagen eliminiert werden konnten, wirkte sich die allmählich zu Ungunsten Canadas verlaufende Weltmarktsituation aus. Sie führte schon um 1930 zu katastrophalen Preisstürzen und drohte die Prärien als Weizenland in Frage zu stellen. Obgleich der zweite Weltkrieg einen kurzen Neuaufschivung brachte, scheint die einstmalige Blüte endgültig vorbei zu sein. Das kommt schon in ciner Skizze der Situation der Prärien um $19+4$ von V. C. Fowke zum Ausdruck: The first world war went far to turn the Prairies into a wheat granary. In this war the big demand has been for bacon, cheese, eggs, beef and lamb, vegetables and dried milk. From 1940 to 1943 Prairie farmers cut wheat acreage 40\% - from 27.750000 to $16730^{\circ} 000 \ldots$ The «expert» always, knew' that growing wheat year after year was a 'badthing,' that what the Prairies needed was more self-sufficiency, more mixed farming 》.

In dieser zuletzt genannten Wirtschaftsform, der Mischfarm, scheint denn auch in naher Zukunft ein Ausweg der Prärieprovinzen aus den in den letzten Jahrzehnten immer wieder auftauchenden Schwierigkeiten gegeben zu sein, dies umsomehr, als man damit bis zu einem gewissen Grade auch das drohende Gespenst der Soil Erosion bannen zu können hofft, das freilich ebensosehr durch einseitige Viehzucht wie durch den Getreidebau heraufbeschworen wurde. Der Weizenbau war im übrigen von den Anfängen der Inkulturnahme der Prärien keineswegs der alleinige Landwirtschaftszweig gewesen. Wenn auch der Weizen stets mehr als die Hälfte des umgebrochenen Ackerlandes - das noch jetzt noch kaum einen Drittel der nutzbaren rund 80 Mio ha Ackerlandes der Prärien ausmacht - beanspruchte, so hatten sich Hafer, Gerste, Roggen, Buchweizen und Lein, sowie in geringerem Maße auch Mais, Soya und Gemüse im Laufe der neueren Zeit doch gleichfalls bemerkenswerte Areale erobert. Dann war mit dem Ackerbauer zumeist auch der Rancher, der Viehzüchter eingewandert, der teilweise sogar noch vor diesem die natürlichen weiten Grasfluren der von ungeheuern Herden von Büffeln und anderem Wild belebten eigentlichen Prärien besetzte und aus ihnen Viehweiden «pastures» und «Ranchen» machte. Und auch die Viehbestände der Prärienprovinzen wuchsen zu bemerkenswerten Zahlen an, so daß von diesen Gebieten ebensogut hätte von Viehzuchtprovinzen gesprochen werden können wie von Getreidekammern. Hatten um die Wende des 19. Jahrhunderts die Anteile der Rinder am canadischen Gesamtbestande kaum $20 \%$ betragen, so stiegen sie bis 1951 bei einem Bestande von 3509000 Köpfen auf nahezu 40 \%, während allerdings die Zahl der Pferde (1910: $860000,1951: 695000)$, der Schweine (1910: 1315 Mio, 1951: 1802 Mio) und der übrigen Viehgruppen anteilmäßig darunter blieb. (Dabei mutet der andauernd geringe Bestand an Schafen im Blick auf die vorwiegend trocken-kühlen Klimaverhältnisse besonders merkwürdig an.). In der Regel ließ sich der Viehzüchter vorwiegend in den aridern Regionen der Prärie, den für den Ackerbauer infolge ihrer stärkern Reliefenergie zum vornherein weniger geeigneten Hochprärien- und Flußlandschaften, also vor allem im SW der Prärienprovinzen nieder. Die beiden Wirtschaftszweige: Weizenbau und Vielizucht existierten mithin von Anfang an mehr oder weniger räumlich getrennt voneinander, so daß Kollisionen kaum auftraten, wenn auch immer die Tendenz vorwaltete, den Viehbetrieb den Farmen, d. h. den Ackerbautrieben einzugliedern. Das scheint insbesondere in der Gegenwart mehr und mehr zu Erfolgen zu führen. Im ganzen gesehen hatte so das Schlagwort "wheat is king 》 schon immer nur relative Geltung für die Prärienprovinzen, wiewohl Gislason Recht hat, wenn er betont: "The prosperity of the region has always depended on the yield and price of this most valuable of all grains grown in temperate climates ». Andrerseits macht er doch mit gleicher Bestimmtheit aufmerksam: The dominant position of wheat as an export commodity in the West makes for a badly balanced and unstable economy. Income from wheat is highly 


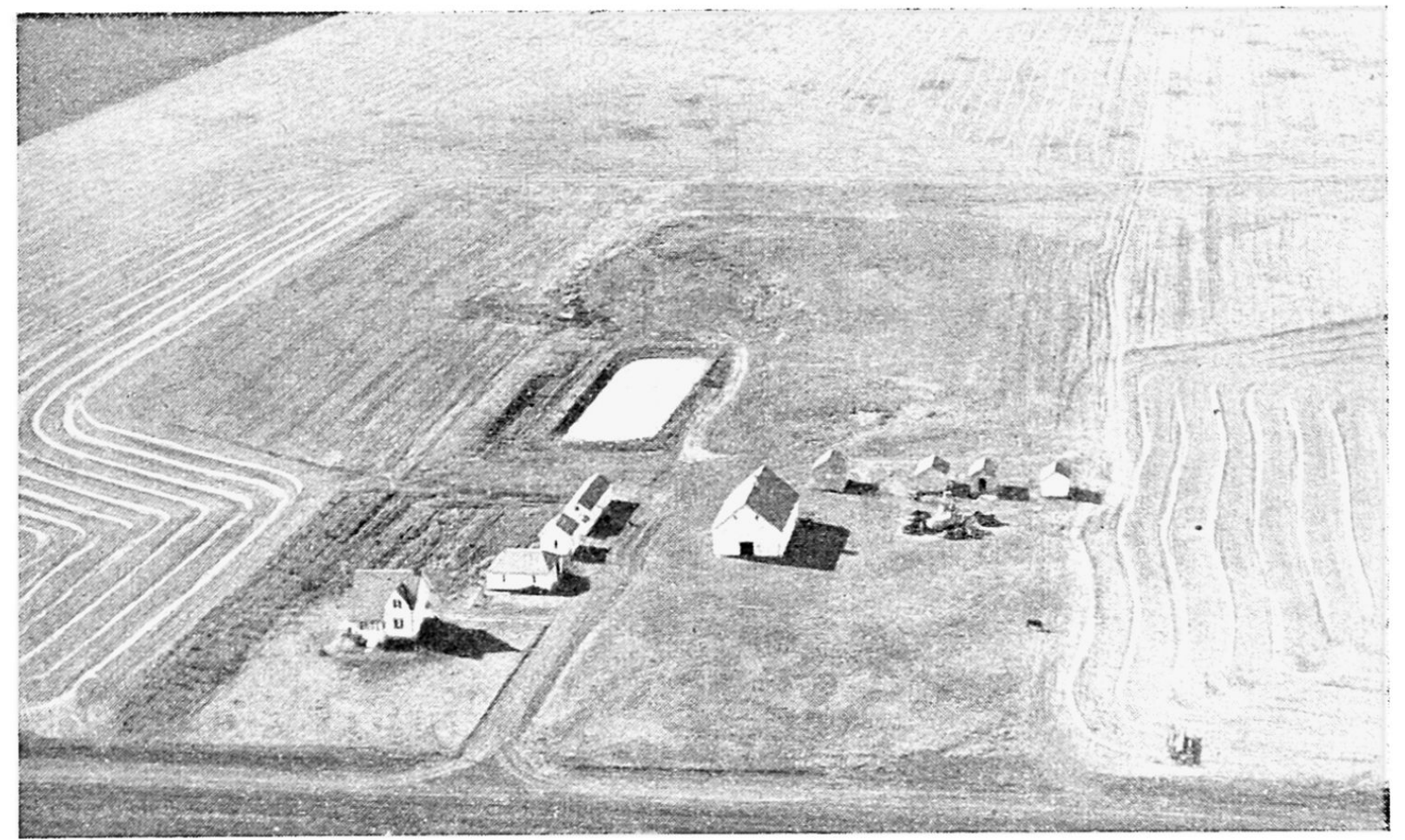

Die Schweizerfarm „Richenberger bei Estlin “ nahe Regina, Südsaskatchewan. Siedlungszentrum inmitten rund 500 acres vorwiegenden Weizenlandes mit Wohnhaus, Wassertank, Gerätehaus (Mitte), Ställen und Kornscheunen, als Symbol der „, bisherigen “ Prärienlandschaft.

variable; yields, grades and world prices are subject to frequent and drastic changes. At the same time the wheat farmer's costs are relatifely fixed. . . . It would therefore seem that greater diversification in farming on the prairies is an necessity $\gg^{2}$. Auf diese Notwendigkeit hatten im übrigen keineswegs nur einsichtsvolle Fachleute hingewiesen. Die Tatsache, daß zwischen 1936 und 1946 ein Rückgang sowohl der Gesamtbevölkerung von 2416000 auf 2363000 als auch der Farmen (1941: 300 000, 1951: knapp 250 000), bedeutete einen Fingerzeig für Regierungen wie Bevölkerung selbst, der klar genug die zu erwartende Zukunftsentwicklung bei einseitiger Landwirtschaft beleuchtete ". Und sie läßt auch U rteile guter ausländischer Kenner wie etwa C. Schotтs verstehen, der 1950 wohl in erster Linie aus der Abnahme der Gesamtbevölkerung und aus dem speziellen Rückgang der (auf die Vergrößerung der Farmen infolge der zunehmenden Mechanisierung zurückgeführten) Landbevölkerung, folgerte, die Prärien hätten «heute bereits den Höhepunkt ihrer Entwicklung überschritten ».

Dies Urteil erscheint nun allerdings bereits heute - nach zwei Jahren - revisionsbedürftig. Nicht nur hat seit 1946 bereits wieder (mit Ausnahme von Saskatchewan, das seither eine weitere Abnahme der Einwohnerschaft von 833000 auf $\$ 32000$ erfuhr) eine aufsteigende Bevölkerungsentwicklung eingesetzt; nicht nur verzeichnet auch der Weizenmarkt eine eher positive Tendenz, es hat sich vielmehr im Zuge der geologischen Detaildurchforschung die Region mehr und

2 Doch bleibt nicht minder auch die These Fowkes beachtenswert: "The mixed farming of the Prairies today is as dependent on world markets as wheat farming ever was! "

3 Dieser offenbar fortdauernde und sich siedlungsgeographisch in starker Wüstungsbildung manifestierende Farm-Rückgang ist „natürlich “ differenzierter zu motivieren als nur durch die - prekäre Marktsituation des Weizens. Mechanisierung und „Industrialisierung “ der Landwirtschaft - deren Betriebsleiter zumeist längst mehr „Fabrikanten “ als Bauern sind, weshalb denn auch der Canadier in der Regel die Landwirtschaft als „, industry “ bezeichnet - sowie Industrialisierung des Landes überhaupt und damit im Zusammenhang stehende soziale und sozialpolitische Umschichtungen usw. bedeuten nicht weniger maßgebliche Hintergründe, wobei es jedoch sehr schwer fällt, eine Priorität der „Ursache“ zu fixieren. 


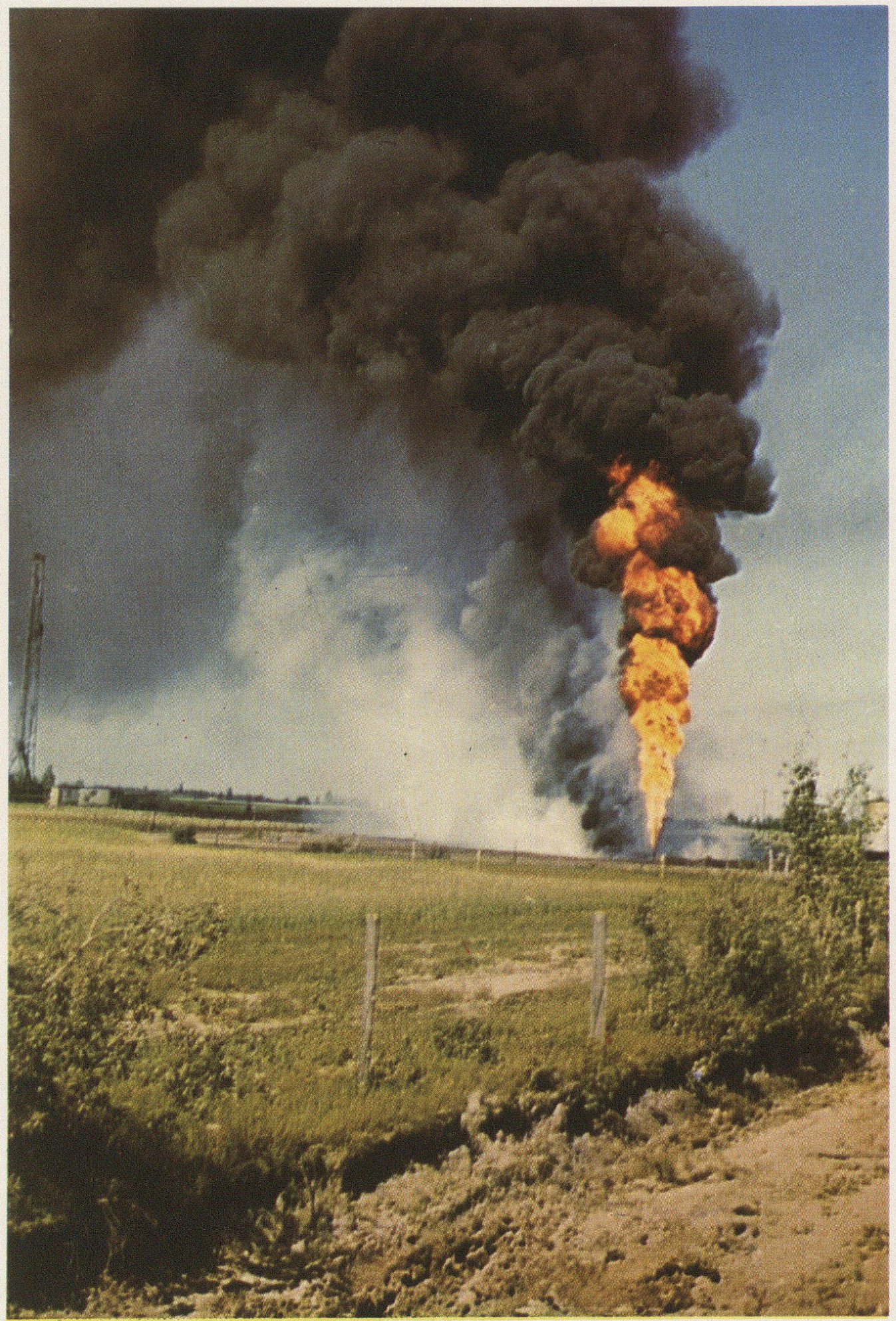

\section{Erdgasflamme in Leduc (Alberta)}

Mit dem Öl gelangen große Mengen Erdgas an die Oberfläche. Ein Teil desselben wird in einer Rohrleitung nach dem benachbarten Edmonton transportiert. Doch große Mengen werden an Ort und Stelle nutzlos verbrannt. Mächtige Feuerfontänen zaubern, vor allem bei Nacht, prächtige Lichteffekte. Doch der beigemengte Ruß verschmutzt die Umgebung und verdrängt den Weizenbau. 


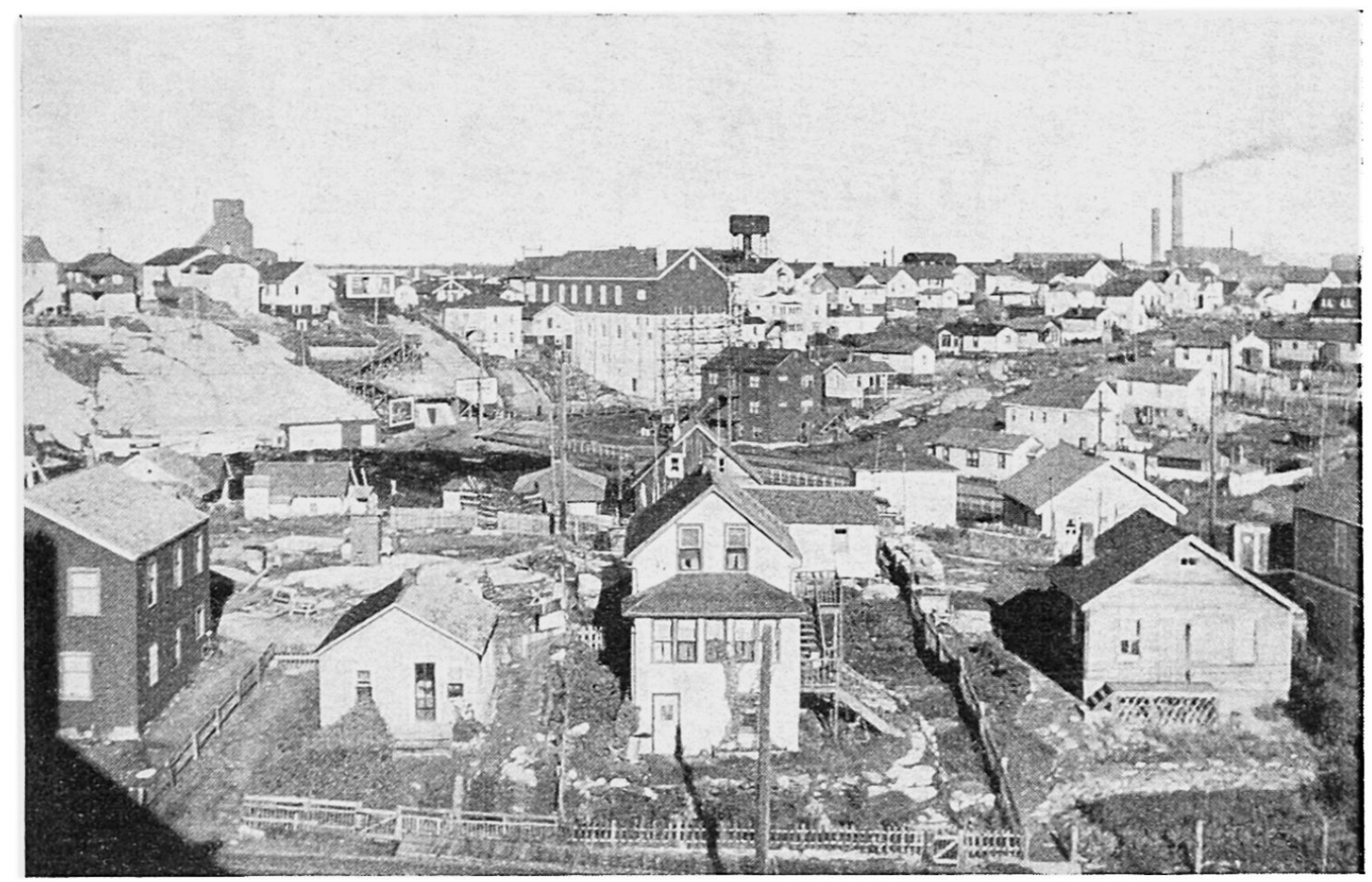

Flin Flon, eine der jüngsten Minenstädte des canadischen Nordens. Blick auf das seit etıva 1930 bestehende Bergbauzentrum. Vorn Häuser von Minenarbeitern, im Hintergrund links der Eingang zum $1300 \mathrm{~m}$ tiefen Hauptschacht, rechts Schmelzwerk, Mitte lutheranische Kirche, deren Unterbau geschäftlichen Zwecken dient. Zwischen Kirche und Schachteingang die „Straße der hundert Treppen“.

Photo H. Bernhard.

mehr als eine Quelle von Mineralreichtümern erwiesen, die einen erneuten Aufschwung in freilich ganz anderer Richtung und mit andern Vorzeichen erhoffen läßt. Und bereits schon sprechen die Zeitungen von einem Erdölboom in Canada, speziell in Alberta, der «die sensationellsten Vorgänge der Vergangenheit in der Ölwirtschaft der benachbarten Vereinigten Staaten — freilich ohne jeden kriminalistischen Einschlag — in Erinnerung bringt» (HöFfDING). Er dürfte wohl im $V$ erlauf der nächsten Jahre nicht nur die Versorgungslage Canadas mit Mineralölprodukten von Grund auf ändern und den jährlichen «Tribut', welchen Canada an die Ölindustrie der Vereinigten Staaten zu entrichten hat, gewaltig vermindern», sondern zusammen mit der aufblühenden Minenwirtschaft im ganzen die Wirtschaftsstruktur der Prärienprovinzen bemerkenswert wandeln.

$\mathrm{Da} \beta$ in Westcanada abbaubare Öl-(und Gas-) felder liegen, - abgesehen davon, $\mathrm{da} \beta$ daneben, namentlich in ihren dem canadischen Schild angehörigen nördlichen 'Ieilen auch andere mineralische Reichtümer zu erwarten sind -, war schon seit längerer Zeit bekannt. Bereits 1914 hatten Prospektoren im Turner Valley, südwestlich der «Fußhügelstadt» Calgary Erdgasquellen entdeckt und daraufhin erste Bohrungen erwirkt, die in der Folge auch auf Petrol fündig wurden. Damit war der Auftakt zu Canadas Erdölindustrie gegeben. Und wenn auch noch über dreißig Jahre verfließen mußten, bis endlich größere Mengen erschlossen wurden, so blieb seitdem die intensive Suche nicht mehr stecken. Der große «Schlag 》 gelang 1947/48 mit der Entwicklung der Erdölfelder bei Leduc und Redwater im Umkreis von Edmonton, der Hauptstadt von Alberta. Dort wurde in devonischen Kalken Öl in so großen Mengen gefunden, daß die Produktion, 1922 erst $14796 \mathrm{Fa} \beta$ (Faß = Barrel $=163.56 \mathrm{l}), 19408495207 \mathrm{Fa} \beta$ und $19476800284 \mathrm{Faß}$ : betragend, in den folgenden Jahren auf 11, 20, 27 und 48 Mio (1951) anstieg. Die gewonnenen Naturgasmengen betrugen 1950: rund 59,9 Mio und 1951: 65 Mio Kubikfuß. 
Canada, das vor 1947 seinen Erdölbedarf nur zum kleinsten Teile aus eigenen Quellen zu decken vermochte, gewann damit plötzlich einen Kraftstoff, der es weitgehend vom Ausland und insbesondere von den USA unabhängig machen wird. Schon 1948 erreichte die Selbstversorgungsrate $25 \%$. Sie hat sich seither, bei einem wachsenden Petrolbedarf, der seit 1951 um 110 Mio Faß ausmachte, weiter gesteigert, und es wird erwartet, daß das Land über kurz oder lang hinsichtlich seines Ölkonsums autark sein werde. Die in den letzten Jahren gemachten erfolgreichen Neubohrungen haben hiefür berechtigte Hoffnungen erweckt. Allein 1950 wurden die Petrolgesellschaften auf 10 neuen Feldern mit 153 Quellen, 1951 sogar auf 33 Feldern mit 780 Quellen fündig. Dabei erweitert sich der Umkreis der Bohrungen und Neuentdeckungen ständig und hat bereits die Provinzgrenze bei Lloydminster (Maidstone, Sask) überschritten, so daß auch Saskatchewan in den Bereich der Ölprospektion geraten ist und erhebliche Aspirationen auf eine künftige Petrolgewinnung hegt.

Die Frage einer Intensivierung der Eigenversorgung ist damit kein Problem der Rohölgewinnung mehr, sondern wesentlich nur noch ein solches der Kapazität der Raffinerien und der Verteilung durch Pipelines. Auch dieses Problem ist inzwischen bereits zu einem nicht geringen Teil gelöst worden, nachdem 1951 die Rohrleitung von Edmonton nach Superior an den Obern See fertiggestellt war und bald eine solche nach Vancouver errichtet sein wird. Die erstere ist $1795 \mathrm{~km}$ lang, hat eine Kapazität von maximal $50000 \mathrm{Fa} ß$ pro Tag, soll jedoch mittelst Pumpen und Vergrößerung der Oeltanks in Superior und Sarnia und durch vermehrten Einsatz von Tankern auf 100000 Faß gebracht werden. Ihr Bau kostete ca. 90 Mill. Dollars. Die im Bau befindliche Pipeline von Edmonton nach Vancouver wird über den Yellowhead $\mathrm{Pa}$ entlang der Canadian National Railway führen und nach ihrer Fertigstellung 1954 täglich $200000 \mathrm{Faß}$ Rohöl im Werte 164 Mill. Dollar nach Vancouver leiten. Diese "Trans-Mountain Pipeline" wird $1200 \mathrm{~km}$ lang sein und etwa 82 Mill. Dollar kosten. Im Rahmen dieser Bauten sind ferner Gasleitungen in den gleichen Richtungen geplant und eine Reihe von Raffinerien im Bereiche der wichtigsten Felder selbst, namentlich um Edmonton, Calgary, Lloydminster und an den Endpunkten der Pipelines betonen schon jetzt den Willen der Canadier, die kostbare Flüssigkeit zum eigenen Nutzen zu verwenden. Dabei ist allerdings festzuhalten, da 3 zwischen 80 und $90 \%$ der Oelfelder in Alberta von Gesellschaften gepachtet sind, die amerikanischen Petrolkonzernen „nahestehen “. So ist die bedeutendste canadische Kompanie, die Imperial Oil Company of Canada, eine Tochtergesellschaft der Standard Oil Co. of New Jersey und kontrolliert vorläufig über die Hälfte der Landesproduktion. Nur etwa $10 \%$ derselben entfallen somit auf Firmen mehr oder weniger rein canadischer Provenienz. Daß ernstlich mit einer konsequenten positiven Entwicklung der Oelproduktion gerechnet wird, belegen die Investitionen, die zwischen 1946 bis 1949 rund 130 Mill. Dollars betrugen, schon für 1950 auf den gleichen Betrag gesteigert wurden und in den kommenden Jahren wohl noch größere Summen annehmen werden.

Wenn nun einstweilen die bestehenden westcanadischen Oelfeder vorherrschend den Bedarf der drei Prärieprovinzen decken und die Verteilung auf größere canadische Gebiete noch in erster Linie eine Aufgabe der Zukunft bleibt, so ist doch die Zeit unschiver abzusehen, da Canada als gleichwertiger Partner der USA auftreten, d. h. vermutlich mit ihnen zusammen den künftigen Bedarf regeln wird. Es ist anzunehmen, daß die Ortsprovinzen Canadas nach wie vor, d. h. auch mit teilweiser Versorgung durch den Mittelwesten, Rohöl aus dem benachbarten Auslande importieren werden, während die Provinz Alberta ihren Überschuß mindestens teilweise in den angrenzenden Staaten der USA absetzen kann.

\section{„THEIR WORK IN MINES AND FACTORIES“}

«For reasons already stated agriculture overshadows all other industries in the Prairie Provinces as a whole. The West is young; its population is small, and distances are great. Under such circumstances manufacturing on any large scale is difficult, for power distribution and transportation are costly. At the same time local markets are limited. On the other hand, these provinces have ample natural resources for extensive industrial development. They have coal, natural gas in abundance along with large resources of oil and water power. What is more, they have a great variety of minerals, in large quantities, and extensive timber resources. Finally they have plenty of raw materials for industrial growth through the processing of vegetable and animal products $\%$. In diesen lapidaren Sätzen hat GISLASON treffend begründet, weshalb bis heute der Weizenbau, «the fun- 
Skizze der Bergbau- und Industriegebiete der westcanadischen Prärie (Alberta) um ro50:

1 Petrolfelder, 2 Erdgasfelder, 3 Raffinerien, 4 Pipelines, 5 Kohlenlager, 6 Nahrungsmittelindustrien, 7 Metallindustrien, 8 Baugewerbe, 9 Keramik, 10 Eisenbahnen, 11 Provinzgrenzen, 12 Staatsgrenzen.

An Kohlen produzierten die Prärieprovinzen (Saskatchewan, Alberta) 1951 mit 9,98 Mio t (1950: $10,3)$ nahezu $53 \%$ der canadischen Erzeugung. Nach Canad. Mining Journal 1952, Nr. 2. 1951 stellten die Prärieprovinzen mit insgesamt $476 \mathrm{Mio}$. Faß Erdöl im Wert von 118,8 Mio. $\$$ gut $99 \%$ der canadischen Produktion: Daran beteiligten sich die Felder von Redwater (23,2 Mio. Faß) undLeduc-Woodbend (13,7 Mio.) zusammen allein mit gut $77 \%$ der Produktion Albertas und mit über $76 \%$ der gesamtcanadischen Erzeugung, während die nächstgrößeren Felder (Turner Valley, Golden Spike, Joseph Lake, Stettler, Lloydminster u. a.) dahinter stark zurückblieben.

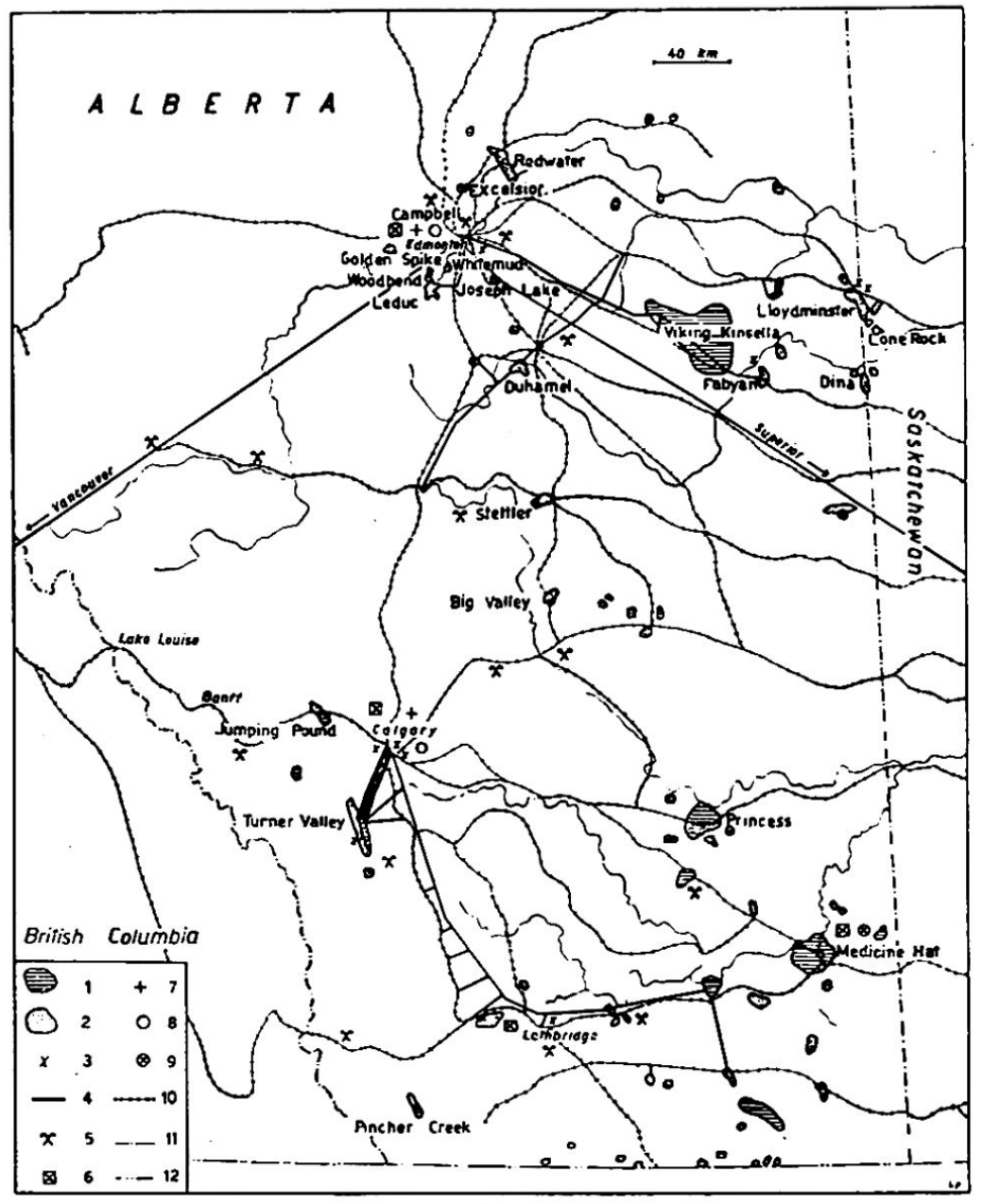

damental, the main industry » des Mittelwestens war und blieb, er hat aber zugleich gezeigt, welch eindeutige, wirkkräftige Impulse einer erfolgreichen Industrialisierung in seiner Natur latent vorhanden sind. $\mathrm{Da}$ die Industrialisierung bereits begonnen hat, haben die knappen Hinweise auf den im Zuge befindlichen Ölboom im Westen der Provinzen anzudeuten vermocht. Daß sie trotz der bestehenden zahlreichen «handicaps》 seit der Konstitution der Provinzen auch schon « rapide Schritte » in Richtung einer Eroberung des Mittelwestens getan hat, vermögen die folgenden Zahlen darzutun, die belegen, da $\beta$ mindestens absolut betrachtet, von einem bemerkensiverten Aufschwung der Industrie gesprochen werden kann:

\begin{tabular}{cccc} 
Jahr & Fabrikbetriebe & Beschäftigte & Bruttoproduktionswert $S$ \\
\hline 1900 & 429 & 6387 & 14892426 \\
1910 & 902 & 27555 & 78794566 \\
1920 & 2134 & 43050 & 306377616 \\
1930 & 2532 & 47835 & 299016538 \\
1939 & 2785 & 43097 & 282418264 \\
1944 & 3509 & 75484 & 780633722 \\
1948 & 3894 & 76699 & 1049654000
\end{tabular}

Seit diesen Erhebungen ist nicht zuletzt unter der Einwirkung der erneuten Rüstungen und der Ölfunde eine weitere Vermehrung der Betriebe sowie der Be-. schäftigten eingetreten, so daß gegenwärtig mit gegen 4500 Fabriken und nahezu 100000 Manufaktur-Arbeitern $\mathrm{zu}$ rechnen ist. Im gleichen Zuge erfolgte .cine Vergrößerung der Betriebe von ehemals knapp 10-15 auf über 20 Arbeiter. Berücksichtigt man, daß in diesen Ziffern weder die Bauindustrie, noch die vielfach mit Minen zusammenhängenden Primärindustrien, die weitgehend «industrialisierten 》 Gewerbe der primären Holzverarbeitung, Fischerei, der Verarbeitung von Agrar- 
produkten enthalten sind, dann tritt die Entfaltung dieses Zweigs der Gesamtwirtschaft in den letzten Jahrzehnten noch betonter hervor. Und wenn er auch weder an eigentliche Industrieländer wie etwa die Schweiz (Zahl der Betriebe 1948: 11528 , der Arbeiter: 531 303) noch an die benachbarten Provinzen oder an ganz Canada (1948: 33447 Betriebe, die Prärieprovinzen also nur gut 11\% derselben, 1,1 Mio Arbeiter, die Prärieprovinzen gut $6 \%$ bei einer Bevölkerungszahl, die immerhin nahezu $17 \%$ der gesamtcanadischen beträgt) heranreicht, so beweist das konstante Anwachsen sowohl des Produktionspotentials wie der Produktionswerte, daß dieser Aufschwung noch stets im Zuge ist.

Mit ihm in deutlichem Zusammenhang steht zweifellos die ausgesprochene Verstädterung, die alle drei Provinzen erfaßt hat, wenn auch die am meisten agrarisch gebliebene Provinz Saskatchewan sich gegenüber den beiden anderen etwas distanzierte. Betrug der Anteil der städtischen bevölkerung an der Gesamteinwohnerschaft 1901 nur $23 \%$, so stieg er 1921 auf 37 und 1951 (letzte Zählung, provisorische Ergebnisse) auf nahezu $49 \%$. Die Verstädterung hat also anscheinend erheblich stärkere Ausmaße angenommen als in Ländern mit ausgedehnterer Industrie (Schweiz: $195036 \%$ ). Die absoluten Zahlen vermittelt die folgende Tabelle:

\begin{tabular}{|c|c|c|c|c|c|c|}
\hline & \multicolumn{2}{|c|}{1901} & \multicolumn{2}{|c|}{1921} & \multicolumn{2}{|c|}{1951} \\
\hline & Landbev. & Stadtbev. & Landbev. & Stadtber. & Landber. & Stadtbev. \\
\hline $\begin{array}{l}\text { Manitoba } \\
\text { Saskatchewan } \\
\text { Alberta } \\
\text { Prärieprovinzen }\end{array}$ & $\begin{array}{r}184775 \\
77013 \\
54489 \\
316277\end{array}$ & $\begin{array}{r}70436 \\
14266 \\
1 S 533 \\
103235\end{array}$ & $\begin{array}{r}348502 \\
538552 \\
365550 \\
1252604\end{array}$ & $\begin{array}{l}261616 \\
218958 \\
222904 \\
703478\end{array}$ & $\begin{array}{r}392112 \\
461045 \\
441313 \\
1294470\end{array}$ & $\begin{array}{r}384429 \\
370681 \\
488188 \\
1143298\end{array}$ \\
\hline
\end{tabular}

Auch im Vergleich mit Gesamtcanada erscheinen die Prärieprovinzen (relativ gesehen) dem Verstädterungsvorgang stärker anheimgefallen zu sein; die relative Zunahme der Stadtbevölkerung betrug in ihnen $26 \%$, in Gesamtcanada nur $24 \%$ (d. h. in Canada nahm die Stadtbevölkerung von 38 auf gut $62 \% \mathrm{zu}$ ).

Wenn freilich die Verhältnisse mit europäischer Statistik beurteilt werden, so bleiben die Zahlen für die städtische Einwohnerschaft erheblich unter den obgenannten Angaben. Denn die in eigentlichen Städten, Siedlungen mit mehr als 10000 Einwohnern lebende Bevölkerung betrug 1951 nur $776743 \mathrm{Köpfe}$ oder nur gut $30 \%$ der Gesamtbevölkerung, wobei sie sich auf 6 Städte zwischen 10 und 50000,2 auf 50-100000 und 3 auf solche über 100000 , Großstädte im engern statistischen Sinne verteilten (Winnipeg mit 236000 bzw. 354000 Einwohner, letztere Zahl innerhalb der sogenannten Metropolitan Area, Edmonton 160000 bzw. 173000 und Calgary 129000 bzw. 139000 Finwohnern).

Daß die naturgemäß dennoch bestehende Verstädterung zum mindesten teilweise mit der Industrialisierung zusammenhängt bezw. mit dieser parallel geht, belegen auch die Relativzahlen der Konzentration der fabrikindustriellen Produktion. Diese war (1946) in Manitoba zu nahezu $89 \%$, in Saskatchewan zu $78 \%$ und in Alberta zu $85 \%$ in den Städten konzentriert. Dabei dürfte dies Verhältnis in Wirklichkeit noch ausgeprägter sein, insofern z. B. gut $80 \%$ der Fabriken von Manitoba ihre Standorte in Greater $W^{\top}$ innipeg haben und eine ähnliche Zentralisation auch für die übrigen Provinzen gilt. Zudem zeigt sich die Tendenz einer zunehmenden Zentralisation auch in den jüngstvergangenen Jahren, wenngleich auch, namentlich im Gefolge der Petrolfunde, daneben in gewissen Gebieten dezentralisierende Tendenzen auftreten. Trotz diesem Entwicklungsstand kann von einer mit extremer Industriekonzentration verbundenen Verstädterung im Grunde kaum gesprochen werden, obgleich dies die Statistiken anscheinend beweisen. Dafür sind die geringen Zahlen der Gesamtbevölkerung ${ }^{4}$, die weiten Distanzen zwischen den städtischen Siediungen, das Fehlen ausgesprochener Großstädte und der weitgehende Gartenstadtcharakter der wenigen «weitläufigen» Städte

\footnotetext{
4 Die mittlere Dichte betrug noch 1951 für die Prärienregien: 1,3 bei sehr geringen provinzialen Unterschieden: Manitoba 1,2, Saskatchewan 1,3, Alberta 1,4 (Canada 1,4).
} 


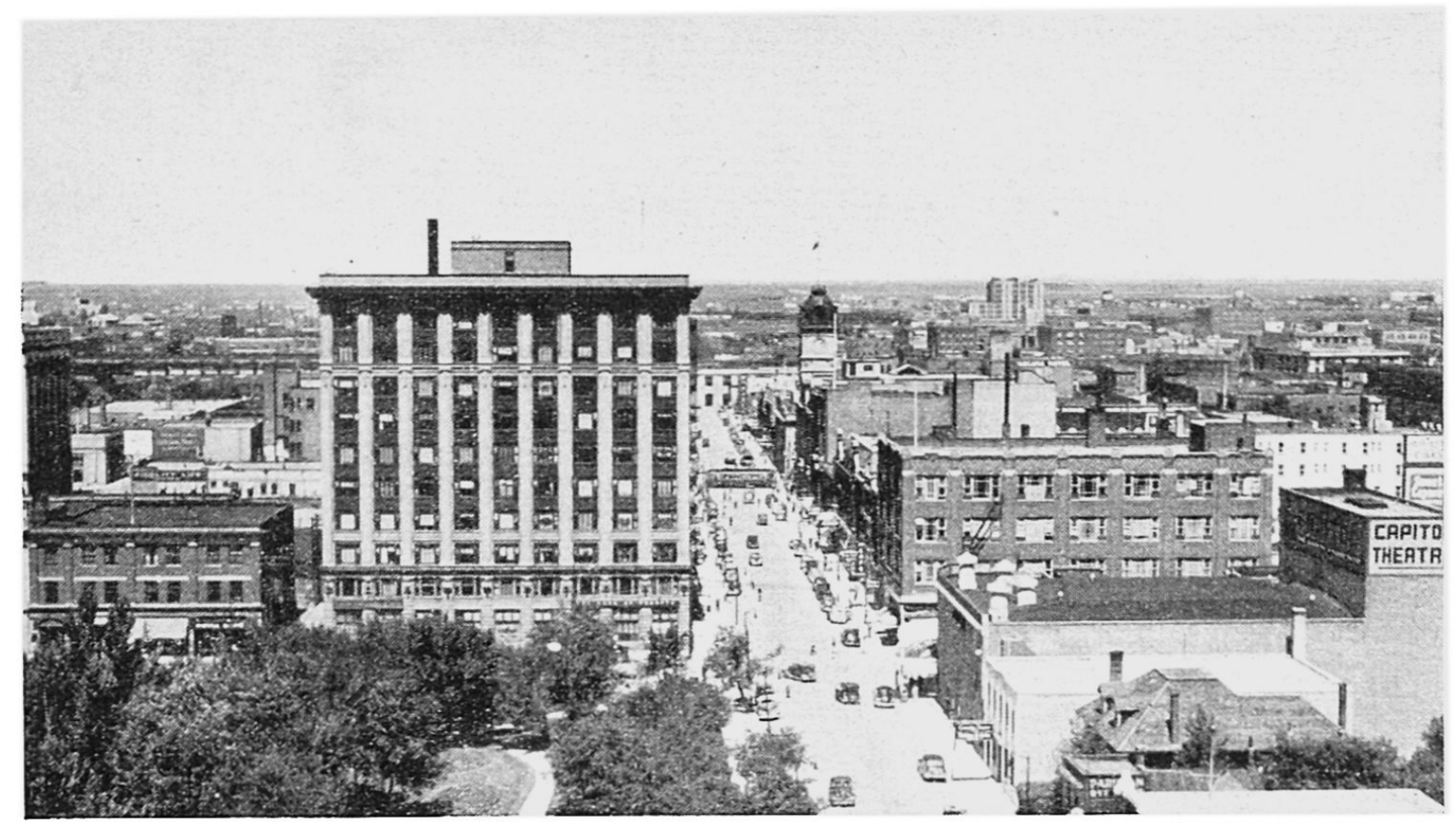

Regina, Hauptstadt von Saskatchewan. Blick auf Scarth Street, City und Stadtrand mit Fabriken der Müllerei-, Metall- und Petrolindustrie. Auch in dieser vor kurzem noch rein ländlich anmutenden, gartenartigen Mittelstadt, der Metropole der ausgesprochensten Agrarregion der Prärienprovinzen, zeichnet sich mehr und mehr der industrielle "Umbruch “ ab, erfaßt jedoch vor allem die suburbane Zone (Suburb).

mit mehr als 100000 Einwohnern die überzeugendsten Gegenbeweise. Dies zeigt die anschließende Tabelle. Immerhin werden für die Zukunft die Provinzen dafür zu sorgen haben, daß Auswüchse wie sie etwa der Osten Canadas, vor allem aber die USA kennen, in ihnen vermieden werden, wobei auch die Wandlungen der Sozialstruktur gerade des letzten Jahrzehnts (absolute und relative Zunahme der Arbeitnehmer, der Fabrikarbeiter [am 2. 3. 1952 standen nach Schätzungen 352000 bäuerlich 'Tätigen bereits 547000 Arbeiter gegenüber] wie der völkischen Heterogenität) keineswegs leichte Probleme stellen.

Be.völkerungsberwegung der Prärieprovinzen und iltrer Stälte über 10000

\begin{tabular}{|c|c|c|c|c|c|c|}
\hline Provinzen/Städte & 1901 & 1926 & 1951 & $\begin{array}{c}\text { Zunahme } 1901 / 51 \\
\text { um das }\end{array}$ & $\begin{array}{l}\text { Industrien } \\
\text { Fabriken }\end{array}$ & $\begin{array}{l}1949 \\
\text { Arbeiter }\end{array}$ \\
\hline Manitoba & 255211 & 639056 & 776541 & 3 fache & 1520 & 41956 \\
\hline Winnipeg & 42340 & 191998 & 235710 & 6 fache & 860 & 28687 \\
\hline St. Boniface & 2019 & 14187 & 26342 & 13 fache & 86 & 4225 \\
\hline Brandon & 5620 & 1644.3 & 20598 & 4 fache & 41 & 814 \\
\hline Flin Flon & - & - & $9899 *$ & - & - & - \\
\hline Saskatchervan & 91279 & 820738 & 831728 & 9 fache & 962 & 10841 \\
\hline Regina & 2249 & 37329 & 71319 & 32 fache & 137 & 2960 \\
\hline Saskatoon & 113 & 31234 & 53268 & 48 fache & 109 & 2524 \\
\hline Moose Jaw & 1158 & 19039 & 24355 & 21 fache & 48 & 1446 \\
\hline Prince Albert & 1785 & 7873 & 17149 & 10 fache & 32 & 896 \\
\hline Alberta & 73022 & 607599 & 939501 & 13 fache & 1685 & 26425 \\
\hline Edmonton & 4176 & 65163 & 159631 & 38 fache & 287 & 8544 \\
\hline Calgary & 4392 & 65513 & 129060 & 29 fache & 276 & 7848 \\
\hline Lethbridge & 2072 & 10893 & 22947 & 12 fache & 41 & 977 \\
\hline Medicine Hat & 1570 & 9536 & 16364 & 10 fache & 33 & 1042 \\
\hline Prärieprovinzen & 319512 & 2067393 & 2547770 & 8 fache & 4167 & 69222 \\
\hline Canada & 5371315 & —** & 14009429 & 3 fache & 35792 & 1171207 \\
\hline
\end{tabular}

* jetzt ca. $11000 \quad * *$ nur Erhebung in den Prärieprovinzen 
$\mathrm{Da}$ vorderhand die Hauptindustrien der Prärieprovinzen entscheidend von der Landwirtschaft - vom Getreidebau, von der Viehwirtschaft (Fleischproduktion) und Milchwirtschaft - abhängen, ist eine ihrer bisherigen Gesamtwirtschaftsstruktur und -entwicklung durchaus entsprechende Situation. An sie schließen sich nach dem Grad der Bedeutung vor allem die Großwerkstätten des Waggon- und Lokomotivbaus, Fabriken zur Erzeugung landwirtschaftlicher Maschinen, Motoren usw., die hauptsächlich um Winnipeg, dem Torweg zu den Prärien und wichtigsten Bahn- und Straßenknotenpunkt, gelagert sind. In raschem Ausbau begriffen sind sodann, wie bereits angedeutet, die Petrolraffinerien. Hinsichtlich der erzeugten Werte an Konsumgütern stehen unzweifelhaft die Betriebe der Fleischverarbeitung (Meat packing plants, Slaughtering Plants usw.) nach wie vor an erster Stelle im Rahmen der drei Provinzen als wirtschaftlicher Einheit, wobei sich ihre Produktionswerte im letzten Jahrzehnt bei jährlichen Summen von 200 bis 250 Mio Dollars auf $20-30 \%$ der Gesamtproduktion beliefen. Dahinter stand die Müllerei mit einem Drittel bis einem $Z_{\text {weitel dieser }}$ Werte bereits wesentlich zurück und noch in weiterem Abstand folgten die übrigen $Z$ weige, die immerhin in zahlreiche Gruppen zerfielen. Daß inskünftig entschiedene Verschiebungen nach Regionen, Branchen und Produktionswerten und insbesondere verstärkte analoge Differenzierungen eintreten werden, zum Teil sogar bereits vor sich gehen, dafür ist nicht nur der Ölboom ein deutlicher Hinweis. Nicht minder bezeugt dies der intensive, teilweise sogar fieberhafte Ausbau der Montanwirtschaft, der in den letzten Jahren mehr und mehr den canadischen Norden einbezieht und zu neuen Bahnbauten, StraBen und Fluglinien geführt hat. Als ein repräsentatives Beispiel dafür darf die "Verlegung» der Sherridon Minen in Mittelmanitoba nach Lynn Lake, rund $200 \mathrm{~km}$ nördlich davon gelten, da sie zeigt, mit welchen Aspekten und welcher Großzügigkeit gearbeitet wird.

Nachdem die genannte Kupfermine der Sherritt Gordon Company in den letzten Jahren offenbar $\mathrm{zu}$ wenig ausgiebig geworden war, andrerseits bedeutende Nickel-Kupferlager in Lynn Lake (und zwar unter ähnlichen geologischen Bedingungen, d. h. als Gemisch von Kupferglanz und Magnetkies in metasedimentären und granitischen Gneisen des Kewatin) gefunden worden waren, beschloß die Kompanie im Jahre 1949 kurzweg die Schließung der Sherridon-Mine. Die bisher erwiesene Reserve in Lynn Lake beläuft 'sich auf 12650000 Tonnen eines Erzes, das einen Wert von rund 175 Mill. Dollars repräsentiert und zu dessen Abbau die Canadian National Railway eine nahezu $240 \mathrm{~km}$ lange Bahn als Anschlußlinie an die Hudson- und Flin Flonbahn in Bau genommen hat. Den besondern Charakter empfängt die neue Minenregion durch die Absicht der Minengesellschaft, an Ort und Stelle das Erz zu verarbeiten, d. h. Fabriken zu errichten, wobei mit einer vorläufigen Aufarbeitung von täglich $2000 \mathrm{mT}$ Erz gerechnet wird. Es sollen sowohl ein hochwertiges Kupfer als ein hochwertiges Nickelkonzentrat an der Mine produziert werden. Ersteres soll sodann nach der Hudsonbay Mine in Flin Flon transportiert und dort weiter verarbeitet werden. Das Nickelkonzentrat ist zum Abtransport nach Fort Saskatchewan (ca. $15 \mathrm{~km} \mathrm{NE} \mathrm{Edmonton)} \mathrm{bestimmt,} \mathrm{wo} \mathrm{eine} \mathrm{Nickelraf-}$ finerie im Bau begriffen ist, die mit Naturgas aus den benachbarten Feldern betrieben werden soll. Die Vollproduktion der Mine wird für das Jahr 1953 erwartet. Kulturlandschaftlich handelt es sich um einen Vorgang der unmittelbaren und völligen Transformation einer Naturregion im Bereich subarktischer "Wildernis" in eine moderne (disjunkte) Industrielandschaft mit Stichbahn, Minen, Fabriken und demgemäß natürlich auch modernen Arbeiter- und Verwaltungswohnbauten, Geschäftsund Gesellschaftsquartieren (voraussichtlich im Stile der canadischen Blockhäuser aber mit allem Komfort, den die durchschnittliche canadische Stadt besitzt) und Sportanlagen, da nur auf Grund solcher "Rekreationsmöglichkeiten " sich Arbeiter in diese menschenleeren Wald- und Felsgebiete antverben lassen.

Dieses Beispiel, das für zahlreiche andere zu stehen vermag, zeigt besser als viele Statistiken, daß gegenwärtig eine eigentliche geologische «Neuentdeckung Canadas» (HöFFDING) im Gange ist, die beinahe täglich zu neuen mehr oder weniger wertvollen Mineralfunden führt und zweifellos der Industrialisierung der Prärieprovinzen starken Vorschub leisten wird. Wenn auch der Anteil ihrer Montanproduktion an derjenigen Canadas noch relativ gering erscheinen mag, so beweist die andauernde Zunahme in den letzten 10 Jahren, daß die Prärien auch in dieser Hinsicht bereits eine bemerkenswerte Entwicklung hinter sich haben. Betrug er nämlich 1940 


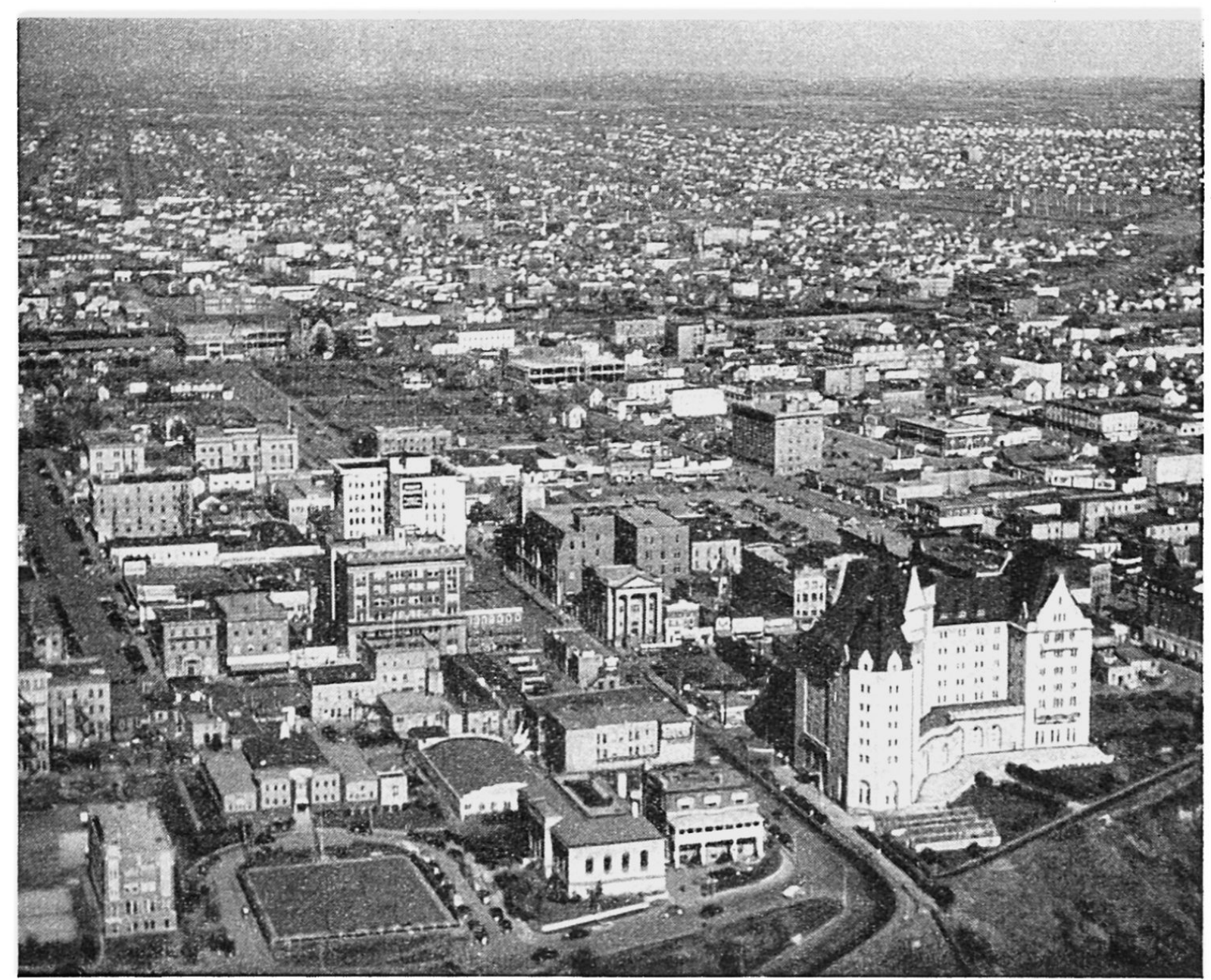

Edmonton, die Hauptstadt von Alberta, Blick auf City, Wohnquartiere und Stadtrand des „Gateway to the North " des Ausgangspunktes der Alaska-Straße und der Minenunternehmungen in NordCanada sowie Zentrum der Erdölfelder. Edmonton hat sich namentlich im Jahrzehnt 1940-1950 wohl am stärksten unter allen canadischen Städten entwickelt, nicht zuletzt dank seines Flugplatzes, der zu den am meisten frequentierten der Erde gehört. Photo Canadian Pacific Railway.

bei einem absoluten Gesamtertrag von 64,4 Mio Dollar $12 \%$, so steigerte er sich 1950, als für 204,4 Mio Dollar Minenprodukte produziert wurden auf $19 \%, 1951$ bei 252,5 Mio Dollars Erlös sogar auf über $20 \%$. Und die Tatsache, daß gegenwärtig gerade im $\mathrm{N}$ der Provinzen erfolgreich nach seltenen Metallen gefahndet wird - in N-Saskatchewan z. B. waren 1951, in der sogenannten Goldfields Area (Athabasca Lake) 15 Uran-Minengesellschaften, in den Black Lake, CharleboisSpreckley Lake und Lac La Ronge Areas deren 5 in Aktion, deren Tätigkeit naturgemäß von einem «dichten Schleier des offiziellen Geheimnisses umhüllt ist 》 bedeutet einen weitern sprechenden Hinweis auf das in der Tat fieberhafte Bemühen, die zweifellos reichen Schätze des Bodens zu heben - und für den Ausbau der Wirtschaft und insbesondere auch der Industrie zu nutzen.

Wenn hier dem in schnellem Flusse befindlichen Geschehen entsprechend nur in Andeutungen auf die jüngste geradezu aufsehenerregende wirtschaftliche Entfaltung der Prärien aufmerksam gemacht werden konnte, so belegt doch gerade das letzte Jahrzehnt, daß die Prärien Canadas einer wirtschaftlichen Zukunft entgegengehen, in der keineswegs mehr Weizen «king》sein wird. Er wird vielmehr sein «Königtum» mit Wirtschaftszweigen zu teilen haben, die bisher unentwickelt schlummerten. Dabei konnte hier nicht einmal auf die bedeutenden Wasserkräfte, die reichen Holzvorräte - und landschaftlichen Schönheiten - der Provinzen gesprochen werden, die für sich bedeutende Kapitalien darstellen. 
So kurz die «canadische » Phase der Kulturlandschaftsgeschichte der Prärien bisher war, so hat sie doch bereits bemerkenswerte Sukzessionswechsel erfahren, die einer genauern Untersuchung wert wären als sie hier geboten werden konnte. Vom Trapperzeitalter über die Perioden der «Trails» und tastenden bodensteten Besiedlung, zur «granary» des Landes, bis hin zur beginnenden Industrialisierung - welche Fülle von Einzelgeschehnissen verbinden sich da zur lebendigen Metamorphose einer Großlandschaft, deren ganzes Sein in die Zukunft weist!

\section{„WORKING TOGE'THER“}

Mit Recht wies Gislasov - und mit ihm zahlreiche andere die jüngste Entfaltung der Prärien schildernden Autoren - daraufhin, daß einer der bezeichnendsten Faktoren des bisherigen Erfolges der energische und umfassende Ausbau kooperativer Organisationen gewesen sei. Zusammenarbeit, korporatives Teamwork das übrigens, bei den «rauhen Milieuverhältnissen» der Prärien verständlich, in die Anfänge des Mittelwestens zurückreicht und etwa in den « Rochdale Pionieren 》 von 1840-50 beispielhafte Vorbilder hat - dürfte aber auch bei den gigantischen Entwicklungsaufgaben der Zukunft das gegebene erfolgversprechende Stimulans sein. Working together, eines der Symbole und zweifellos leuchtenden Sinnbilder des Westens von Canada, kann freilich kaum nur Gemeinschaftswerk innerhalb einzelner Wirtschaftszweige und -richtungen, innerhalb der Fabrikunternehmungen, der Irrigations- und Integralmeliorationswerke, der Absatzorganisationen usw. bedeuten. Es muß freiheitliche Planung im weitesten Sinne des Wortes sein: Planung und Pflege nicht nur der menschlichen Gesellschaft, sondern des Landschaftsganzen und das heißt: Koordination aller Interessen mit dem Ziele harmonischer Kulturlandschaftsgestaltung, die allein dann des Menschen. Existenz im vollen Sinne gewährleistet, wenn er sich nicht nur seiner Ansprüche bewußt bleibt, sondern auch diejenigen der - scheinbar von ihm beherrschten - Natur berücksichtigt. Die Westerner haben in diesem Zusammenhang seit längerem schon großzügige Stadtplanungen in die Wege geleitet (so für Winnipeg, für Reginà, für Edmonton, Calgary u. a.), deren Konzeptionen sich mit besten europäischen und US-amerikanischen Beispielen messen dürfen, wenn auch der Städtebau hinter den gesteckten Zeilen teilweise noch zurückgeblieben sein mag. Sie haben auch Reservate, Naturschutzpärke in einem Ausina $B$ und von einer Anziehungskraft, die alle Achtung abzwingen: die Gesamtfläche der National- und Provinzialpärke beträgt zur Zeit gut $7.6000 \mathrm{~km}^{2}$, ein Areal, das über $66 \%$ der canadischen Reservate beansprucht. Im Grunde geht es bei dieser Forderung nach umfassender Zusammenarbeit, aber nicht um Städteplanung und Schaffung und Erhaltung von Naturschutzgebieten allein, die trotz ihrer respektabeln Größe doch nur als winzige Inseln im unabsehbaren Meer der Wälder, Fels- und Seengebiete, der wundervollen Täler und Hochgebirgslandschaften zerstreut sind. Es geht vielmehr um die Erhaltung und Pflege der Gesamtlandschaft - die teilweise bereits, so in den Weide- und Weizengebieten durch SoilErosion und künstliche Versteppung bedroht - entscheidender Garant der kulturellen Zukunft des Mittelwestens ist.

Noch besitzen die Westerner also gewaltige, reiche, jungfräuliche Räume, prachtvolle Landschaften urwüchsigsten Gepräges. Sie haben damit, wie wenige andere Völker, alle Möglichkeiten, an ihnen sich schulend sich ihr Schicksal selbst durch kluge Landschaftspolitik, -forschung, -gestaltung und -nutzung $\mathrm{zu}$ ihrem eigenen Vorteil zu lenken. Es ist $z u$ hoffen, daß sie bei ihrem optimistischen zivilisatorischen Vorwärtsdrängen dessen eingedenk bleiben, daß «they not solve themselves», daß es zur Erfüllung ihrer Wünsche nicht nur «the courage to experiment» und nicht nur die Meisterung der «handicaps of geography » braucht, sondern daß es vor allen Dingen den Sinn für das Eingeordnetsein des Menschen in den Rahmen einer 


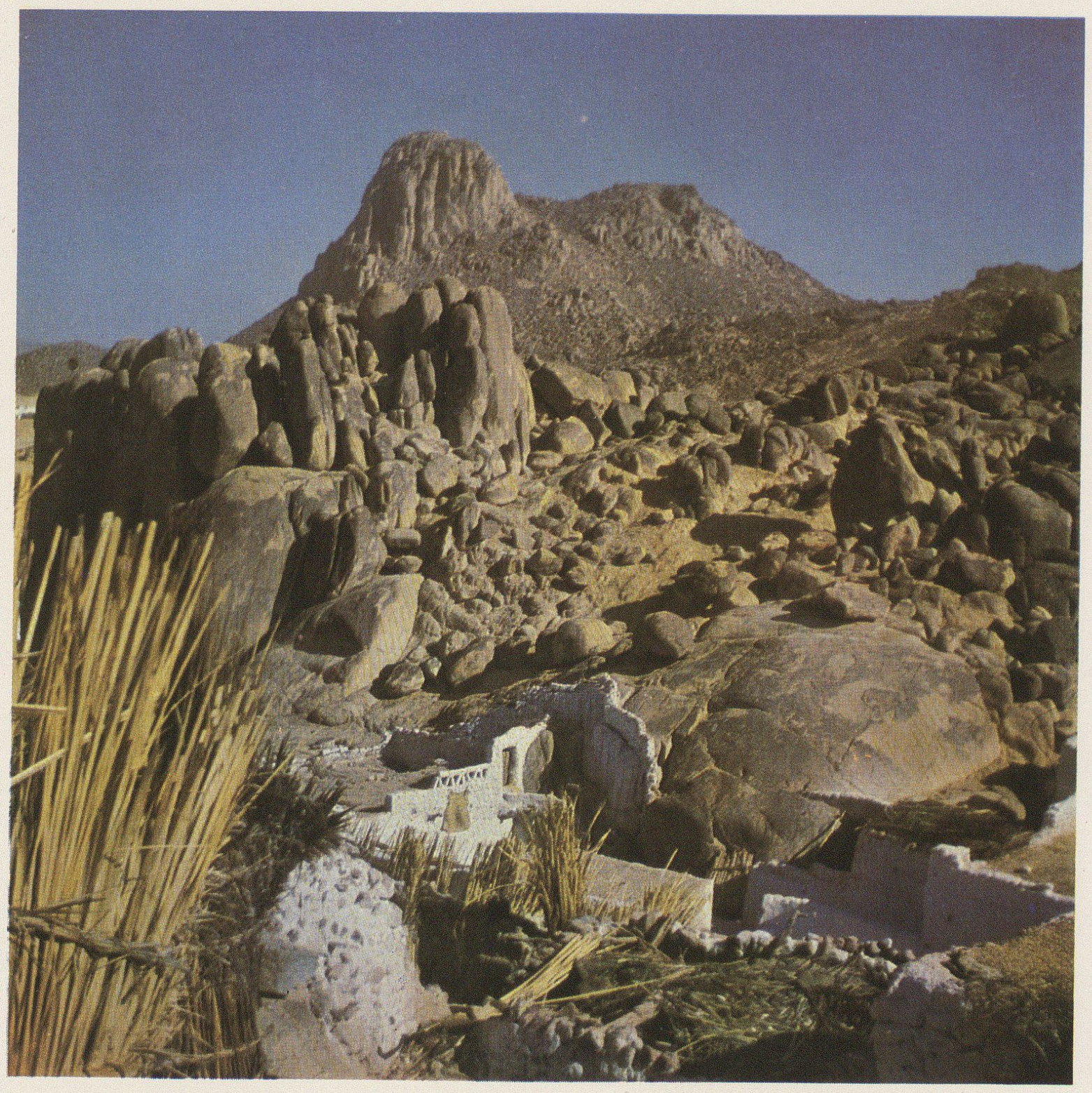

Abgeschliffene Granite und Basalte im abgelegenen Tale von Djanet, irgendwo im Tassili des Ajjer im Wunderland der südlichen Sahara. 
nicht minder wirksamen Natur zu betätigen gilt. Mit dessen Praktizierung nur wird, auch bei zunehmender Industrialisierung, wie Gislason sicher zu Recht betont «prosperity endure for it will rest not on the fortuitous advantage of location, but on their solid material resources and the vigourous enterprising spirit of their people».

\section{BENÜTZTE QUELLEN}

Canada Year Book. Ottawa $1920 \mathrm{ff}$. - Canada. Ottawa $1940 \mathrm{ff}$. - Canadian Mining Journal. Annual Review of Canadas Mineral Industries. Quebec $1879 \mathrm{ff}$. - Eichmerer, M.: Die canadische Prärie als Wirtschaftsraum. Amerikanische Landschaft. Berlin 1934, 129-234 (umfangreiche Bibliographie). - Fowke, V. C.: Prairie Provinces. Ottawa 1944. - Gislason, I.: Prairie Panorama. Calgary 1948. - HarRington, L.: Tractor Trails in Manitoba. Canad. Geogr. Journal 38, 1949, 7177. - Höfrding, W.: Canada, eine Wirtschaftsmacht im Aufstieg. Neue Zürcher Zeitung 170, 1950, Nr. 2661, 2698, 2711, 2730, 2740, 2775, 2794, 2813, 2840. - HolBrook, E. M.: Oil from the Earth. Canad. Geogr. J. 39, 1949, 135-144. - HookE, A. J.: Alberta-Nature's Treasure House. Canad. Geogr. J. 35, 1947, 155-177. - Macfarlane, R. O. : Manitoba. Canad. Geogr. J. 35, 1947, 124-152. - Mackie, V. J.: Manitoba, Province of Industry. Canad. Geogr. J.41, 1920, 167-181. Manufactoring Industries in the Prairie Provinces. Winnipeg 1944. - SPENCE, G.: Water for the Prairies. Canad. Geogr. J. 44, 1952, 48-57. - Schotr, C.: Die canadischen Prärien, Geogr. Rundschau 2, 1950, 1-6. - WRIGHT, J.: Saskatchewan. Canad. Geogr. J. 34, 1947, 109-136. - Ders. : Co-operative Farming in Saskatchewan. Canad. Geogr. J. 39, 1949, 68-90. - The Financial Post. Overseas Edition. Montreal-Toronto-London $1950 \mathrm{ff}$. (insbes. Sondernummer vom 1.3.1951: This is Canada 1951). - Business Year Book. 1952. Montreal-Toronto.

Es ist dem Verfasser Bedürfnis, verschiedenen Persönlichkeiten und Stellen für die freundliche und wertvolle Unterstützung bei der Beschaffung von Grundlagen zu danken, so den Gesandtschaften von Canada (in Bern) und der Schweiz (Ottawa), insbesondere den Herren Minister Dr. V. NEF und Gesandtschaftssekretär Bisang, Prof. Dr. Carl FÆssLeR, Quebec, Dr. J. W. WaTson, Direktor des geographischen Dienstes, des Dept. of Mines and Resources, Ottawa und Dr. A. HuBER, Forstingenieur, Zürich. Herrn Prof. Dr.H. Bernhard danke ich insbesondere für sein Farbbild*, Frl. H. Lamprecht, Assistentin am Geogr. Institut ETH, für die Reinzeichnung der Kartenskizzen.

\section{TRANSFORMATION DES PRAIRIES CANADIENNES PAR L'INDUSTRIE}

Les Prairies du Centre-Ouest du Canada passaient jusqu’ici pour le type de contrées agricoles et surtout de grandes terres à blé, dont la production en froment égalait les $80-95 \%$ de celle du pays tout entier: c'est à cause d'elles que le Canada était considéré dans le monde comme le pays du froment. Mais, dans ces dernières années, se dessine, surtout dans l'Alberta, la plus occidentale des provinces, un rapide changement, dû surtout à la découverte de pétrole et de gaz naturel. L'importance de cette révélation se mesure au fait que le Canada, jusqu'il y a peu d'années, ne couvrait que quelques pour-cent de ses besoins en pétrole, tandis que depuis la mise en exploitation des gisements de la Turner Valley, à Leduc et à Redwater, sa production représente $25 \%$ de sa consommation, montée de 8 à 48 millions de barils (1947-51). Quoiqu'il n'y ait aucune preuve que le Canada en vienne à se suffire à lui-même en ce domaine, cependant les formidables investissements (1950: 150 millions de dollars) prouvent que les cercles compétents ont des espérances sérieusement fondées. En tout cas il en résulte que le Centre-Ouest du Canada, en grande partie tout au moins, semble devoir se transformer en une région industrielle.

\section{L'INDUSTRIALIZZAZIONE DELLE PRATERIE CANADESI}

Le praterie canadesi del medio ponente rappresentarono fino a poco tempo fa l'esempio più spiccato di paesaggio agricolo, coltivato a cereali. La produzione di grano ammontava agli $80-95 \%$ della produzione complessiva del paese contribuendo cosi decisamente alla potenza agricola del Canadà nel marcato mondiale dei cereali. In questi ultimi anni si nota di contro una profonda mutazione del paesaggio agricolo, in modo particolare nella provincia occidentale di Alberta, che ha avuto inizio con la scoperta dei giacimenti di petrolio e di gas. L'importanza di queste scoperte si rivela chiaramente dal seguente fatto: fino a pochi anni or sono il Canadà riusciva a malapena a coprire pochi $\%$ del suo fabbisogno di petrolio, mentre dopo la scoperta delle sorgenti nella Turner Valley, nel Leduc e sul Redwater, vi contribuisce con il $25 \%$ (circa 8 mil. su 48 mil. di berili nel periodo 1947-51). Pur non esistendo la garanzia che il Canadà possa arrivare ad una autarchia completa per il petrolio, l'aver investito ingenti capitali (1950: $150 \mathrm{Mil}$. \$) dimostra che nei circoli interessati sussistono queste speranze. Si è cosi iniziata una profonda trasformazione del medio ponente dei Canadà, trasformazione che tende, almeno per grandi zone, a un paesaggio industriale.

* Aus dem im Verlag Kümmerly \& Frey erscheinenden Buch: Bernhard/Winkler: $A$ mari usque ad mare, Canada zwischen gestern und morgen. 